\title{
Electromagnetic Radiation Hazards on Cellular Bioelectricity of Rats' Blood
}

\author{
Alaa M. Khalil \\ Basic Sciences Department, Faculty of Engineering, Pharos University in Alexandria, Egypt
}

\section{ARTICLE INFO \\ Article history: \\ Received: $18^{\text {th }}$ Oct. 2020 \\ Accepted: 14th Apr. 2021}

\section{Keywords:}

Radiations,

Electric Field,

Magnetic Field,

High Energy Photons,

Blood, Conductivity,

Permittivity,

Dielectric Loss,

Blood Minerals.

\begin{abstract}
The effect of exposure to electric field of $4 \mathrm{kV} / \mathrm{m}-50 \mathrm{~Hz}$, magnetic field of $0.3 \mathrm{mT}-50 \mathrm{~Hz}$ and high energy photons of $\left(6 \mathrm{MeV} ; 0.002 \mathrm{~A}^{\circ}-1 \mathrm{rad}\right)$ on rats' blood bioelectricity were studied. The electrical conductivity, relative permittivity and dielectric loss factors were monitored in the frequency range of $42 \mathrm{~Hz}-8 \mathrm{MHz}$ for blood samples collected directly after exposure and 45 days' post exposure. Moreover, the exposure effect was studied on the blood minerals (Sodium, Calcium, Phosphate and Potassium) and protein concentrations. Our findings illuminated the maximum shift in conductivity at $5 \mathrm{MHz}$ by $62 \%$ and in permittivity by 23 folds higher than control for samples collected after exposure to magnetic fields (30days-8h/day). Then it is followed by samples collected after exposure to high energy photons as conductivity changed by $46 \%$ and permittivity by 8 folds higher than control. On the other hand, maximum deteriorative impact of exposure in minerals was shown for samples exposed to high energy photons, as it indicated the percentage of change in relative to control by $13 \%, 17 \%$ and $5 \%$ for phosphate, calcium and potassium respectively. No improvement was shown for samples collected after 45 days' post exposure. The exposure to magnetic field has a higher effect than the exposure to electric field, even at thesame exposure periods. The effect of exposure to non-ionizing radiations should take same concerns as ionizing radiation. Full blood analysis should including dielectric characteristics as parameters indicate possible cellular injuries. There is a need for more work to study the possible health consequences due to exposure to such non-ionizing radiations and to set new exposure limits.
\end{abstract}

\section{INTRODUCTION}

The electromagnetic environment consists of natural radiation (geomagnetic field, intense solar activity, thunderstorms) and man-made electromagnetic fields (EMF) which includes (factories, transmission power lines, electric appliances at work and home, and medical treatments. The biological effects of these radiation on human health have become the subject of important and persistent public debate. The association between extremely-low frequency (ELF) fields and the risk of blood disease is not clear yet according to the assessment conclusions of World Health Organization (WHO's) international EMF project [1], So still there is a need for many researches to be done about this mystery. The accurate knowledge of the dielectric properties of blood is essential for deciding the limiting values for electromagnetic pollution [2]. The dielectric blood coagulometry may be a useful method for measuring blood clotting and could provide the detailed assessment for the status of anticoagulant therapy [3]. Recently, a study of the dielectric properties of the total serum proteins of the exposed mice to an electric field $(6 \mathrm{kV} / \mathrm{m}-50 \mathrm{~Hz})$ for periods 30,45 and 60 days and for 30 days' post exposure was done. The results indicated that the electric field decreased the permittivity value of the serum proteins and increased its conductivity. Also, the intensity of the absorption spectral bands of the serum proteins of samples collected from the exposed mice showed a significant decrease. However, these findings assured that ELF EMF influence on the biological properties of the cell's membrane protein [4]. Moreover, ELF EF induces electrical potentials, giving rise to resultant current flow in aqueous medium that surrounded the living cells. A small fraction of this induced current penetrates the cell surface because of the membranes of these cells form a dielectric barrier to the passage current in these frequency range. This induced per-cellular current produces electrochemical alterations in the cell membrane surface and sends signals across the 
cell membrane carrier that produces changes in physiological functions [5]. These changes of cell functions may lead to the alterations of resonating metabolic processes of the cell or destroy it, leading finally to the cell death [6]. For this reason, there are many studies aiming at using the low frequency electric field in the treatment of tumor cells.

In addition, there are many studies of the biological effects of magnetic fields at the tissues and cellular levels [7-13]. The electric conductivity of blood serum protein of exposed mice increases due to the changes in the dipole's orientation of their membrane components, which leads to conformational changes in the membrane structure. The dielectric constant values decrease as a function of changes in the dipole moment of the macromolecules which depends on the center of mass of the charge distribution and the molecules radius [7]. The interactions of the electric field and protein molecules cause rearrangement of protein charge distribution and changing its properties. This is in agreement with Mccammon who reported that the variation in the surface charge may cause the enzyme receptors to be more sensitive to potential changes [8].

In addition, the conductivity of red blood cells was sensitive to ionizing radiation, and it is increased by the presence of hemoglobin molecule. The change in conductivity reflects the permeability damage of the cell membrane with the subsequent loss of ions, electrolytes and intracellular components [14]. It altered the relative permittivity and dielectric loss which reflects the possibility that some changes may have happened in the protein of the cell membrane $[15,16]$. Several studies have been performed on the effect of high energy ionizing radiation on red blood cells (RBCs). They showed that lipid peroxidation, cross linking in membrane proteins and change in the membrane permeability will happen after the exposure to gamma radiation [17]. To this end, other studies reported that the exposure of red blood cells (RBCs) to ionizing radiation might cause the loss of potassium and a gain of both sodium and calcium. Former research works stated that ionizing radiation can change the metabolism or altered the Adenosine Triphosphate (ATP)-base activity and may lead to a loss of membrane sulphydryl groups [17]. Recently main concerns were drawn to the health consequences of magnetic fields and ionizing radiation and very limited researches were interested in the hazardous effects of electric fields. Moreover, the effects of exposure to electric and magnetic fields on blood bioelectricity are still unclear, not yet been fully elucidated and a need for more researches to evaluate the exposure impacts on blood minerals became a must. Many researchers monitored the dielectric characteristics of blood cells as biomarker to its vitality and its metabolic activities. Grapping the exposure effects due to magnetic, electric and high energy photons on blood dielectric characteristics and blood mineral concentrations may illuminate new insights about such fields. The present work it has been devoted to study the exposure effect of ELF-EMF as non-ionizing radiation model and high energy photons as ionizing radiation model on the blood dielectric characteristics. Moreover, the dielectric characteristics and biochemical measurements were studied directly after exposure and also after 45 days post exposure.

\section{MATERIALS AND METHODS}

In the present work 150 male albino rats weighing an average of $170 \pm 15 \mathrm{~g}$, were divided into 6-groups. Two groups of 40 rats/each were exposed to an electric field $(4 \mathrm{kV} / \mathrm{m}, 50 \mathrm{~Hz}-8 \mathrm{~h} / \mathrm{d})$ successively during one and four weeks. One group of 20 rats was immediately sacrificed after its exposure period, another group of 20 rats were left to survive and housed at normal environmental conditions similar to the control group and sacrificed after 45 days' post exposure period. Similar to the former direct and delay sacrificed subgroups, two groups of 40 rats were exposed to magnetic field $(0.3 \mathrm{mT}$, $50 \mathrm{~Hz}-8 \mathrm{~h} / \mathrm{d}$ ) successively during one and four weeks. One group of 20 rats was exposed to high energy photon (6 MeV; $0.002 \mathrm{~A}^{\mathrm{o}}-1 \mathrm{rad}$ ) emitted from a linear accelerator (LINAC) at Faculty of Medicine, Alexandria University, Egypt. Similarly, 10 rats were immediately sacrificed after exposure and the other 10 rats were left to survive normally and sacrificed after 45 days' post exposure. For each exposure condition 5 rats were kept without receiving any treatment and left in normal environmental housing whereas it has been sacrificed at the end of the treatment condition. It is worthy to state that the normal environment was adapted inside the lab at room temperature at an average of $25{ }^{\circ} \mathrm{C}$, and lighting condition were natural light from large windows during the day and complete darkness during the night.

Exposure to the electric field was achieved by using mains of output voltage $4 \pm 0.2 \mathrm{kV}$, applied through two Al electrodes connected at the two sides of Perspex chamber. The animals were kept inside the chamber dimensions $100 \times 30 \times 35 \mathrm{~cm}^{3}$ during the exposure period whereas the $\mathrm{Al}$ electrodes were covered by front fixed Perspex sheets of similar dimensions. It should be noticed that the Perspex material has a negligible effect on the field uniformity as reported elsewhere [18]. It is 
important to confirm that the reading of the measured electric field inside the chamber was $4 \mathrm{kV} / \mathrm{m}$.

The exposure to magnetic field was performed using magnetic unit consisting of four solenoid coils of 48 turns and a length of $63 \mathrm{~cm}$ (coils were wrapped by using electrically insulated copper wires of $2 \mathrm{~mm}$ diameter). A magnetic unit of $0.3 \mathrm{mT}$ was used through large cylindrical chamber of dimensions $100 \mathrm{~cm}$ long and $60 \mathrm{~cm}$ wide. The magnetic unit was connected in series with the $50-\mathrm{Hz}$ power supply and ammeter reading is fixed at $0.8 \mathrm{~A}$. The animals were housed inside its Perspex cages of dimensions $30 \times 40 \times 35 \mathrm{~cm}^{3}$ on a shelf inside the central axis of the cylindrical chamber. The exposure of animals to high energy photon (6 MeV; $\left.0.002 \mathrm{~A}^{\mathrm{o}}-1 \mathrm{rad}\right)$ was acquired by placing the animals where the Elekta Linear accelerator (LINAC) output was adjusted to give collimator and couch angles of $0^{\circ}$ and source to surface distance (SSD) of $100 \mathrm{~cm}$. Immediately after exposure all animals were sacrificed, heart punctured and blood samples were collected. The dielectric properties of blood samples were measured by the use of a two-electrode technique configured with an impedance LCR meter (HIOKI 3536, Japan). The electrical conductivity, relative permittivity and dielectric loss factor were scanned for blood samples in the frequency range of $42 \mathrm{~Hz}$ to $8 \mathrm{MHz}$. Moreover, the blood minerals (Sodium, Calcium, Phosphate and Potassium) and blood proteins were measured as adopted by R. J. Henry; 1974 [19] and OH Lowry; 1951 [20].

\section{RESULTS AND DISCUSSION}

The obtained dielectric characteristics overall tested blood samples under the application of different treatments showed remarkable bioelectrical alterations. The dielectric characteristics were obtained by measuring its bioelectric relaxations under the effect of external alternating electric signals in the frequency range of $42 \mathrm{~Hz}$ to $8 \mathrm{MHz}$. The dielectric parameters of blood samples (electrical conductivity, relative permittivity and dielectric loss) were studied as a function of frequency and hence the relaxation curves were obtained. In addition, blood minerals and protein concentrations were measured for exposed and delayed post exposure samples and compared to control ones. Figure (1) shows the conductivity curves of blood samples collected directly after exposure as a function of the frequency. The obtained curves showed a similar plateau shape as the control one, whereas it has different order according to the effect of exposure. The maximum depression of conductivity was obtained for samples collected after exposure to MF-30days followed in depression for samples collected after exposure to high energy photons. On the other hand, samples collected after exposure to EF-7days, did not show a remarkable depression as compared to the other groups where the longer exposure period EF-30days indicated significant shift to lower conductivity values.

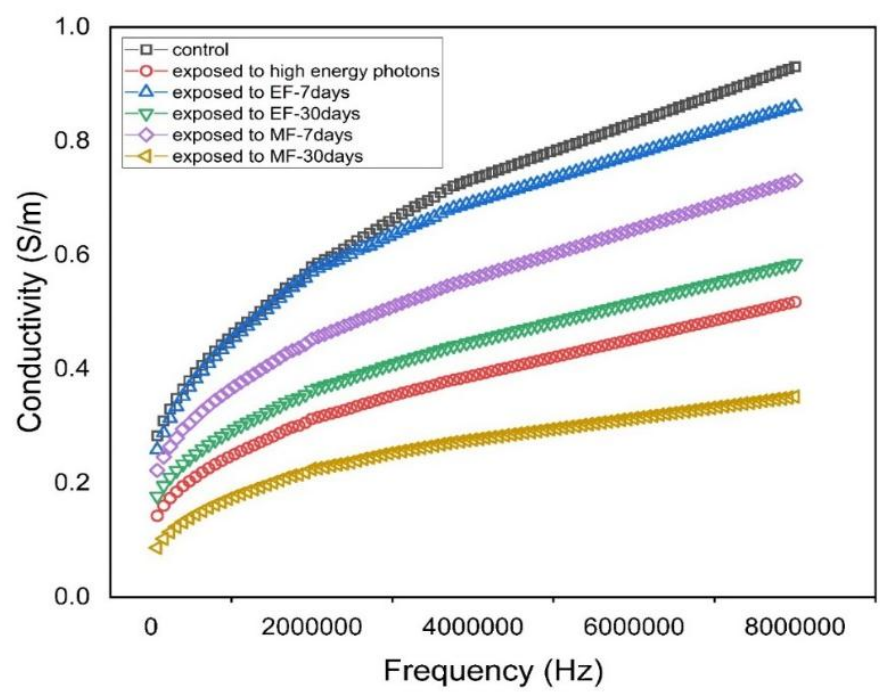

Fig. (1): The conductivity curves of blood samples collected directly after exposure

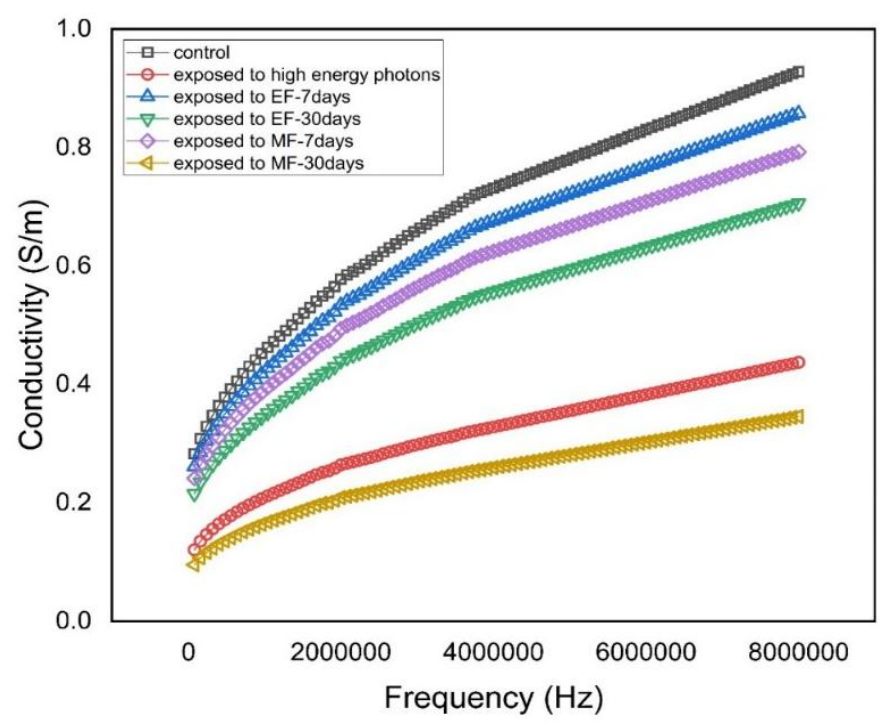

Fig. (2): The conductivity curves of blood samples collected after 45-days post exposure

Pronounced shifts to low values in the conductivity were monitored as shown in Figure (2); for collected samples after 45-days post exposure to MF-30days and high energy photons. The former findings support that the new blood generation didn't show an enhancement and did not fully recovered even after being left 45-days post exposure. On the other hand, the other collected blood samples after 45-days post exposure to EF-7days, 30days and MF-7days indicated an improvement to come closer to the control values. 
It should be noted that the screening of conductivity values over the frequency range is in association with the relative permittivity of the samples and it shows inverse proportionality. Figure (3) shows the relaxation curves of relative permittivity for blood samples collected directly after exposure in comparison with control ones. The obtained relaxations are in a the same manner as the conductivity; the maximum shift in the permittivity for samples collected after exposure to MF-30days and followed by samples collected after exposure to high energy photons. It is worthy to state that the shift in the permittivity values could be remarkably shown in the range of frequency less than $4 \mathrm{MHz}$ as shown in the magnified upper side figure. Figure (4) shows the relaxation curves of blood samples collected after 45-days post exposure as function of the frequency. As aforementioned in the conductivity results the obtained relaxations of blood samples collected after 45-days post exposure to 30MF-30days and high energy photons didn't show amendment on its bioelectricity even for the new blood generation.

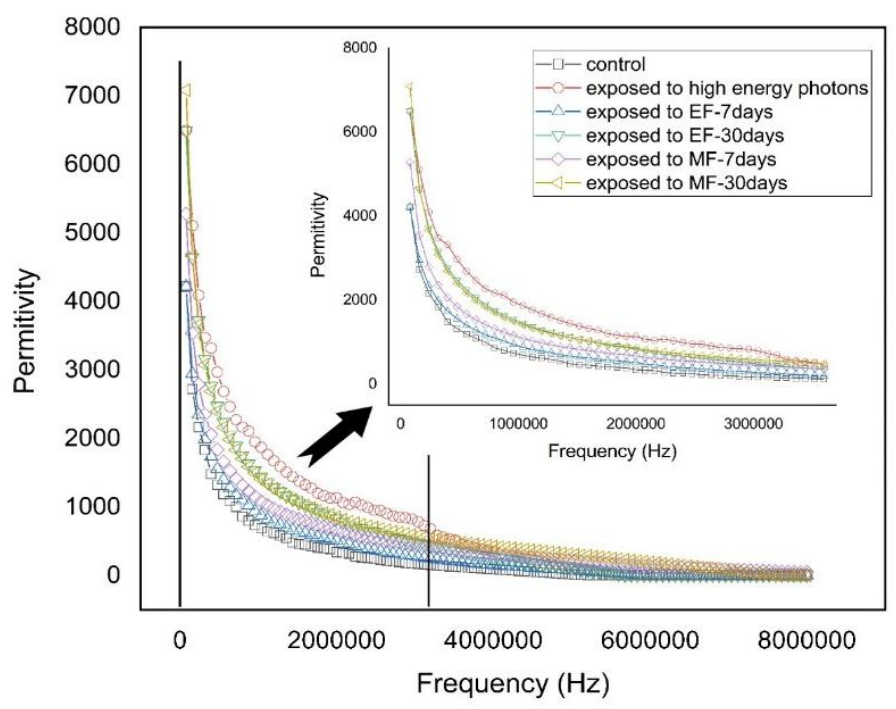

Fig. (3): The relative permittivity relaxation curves of blood samples collected directly after exposure

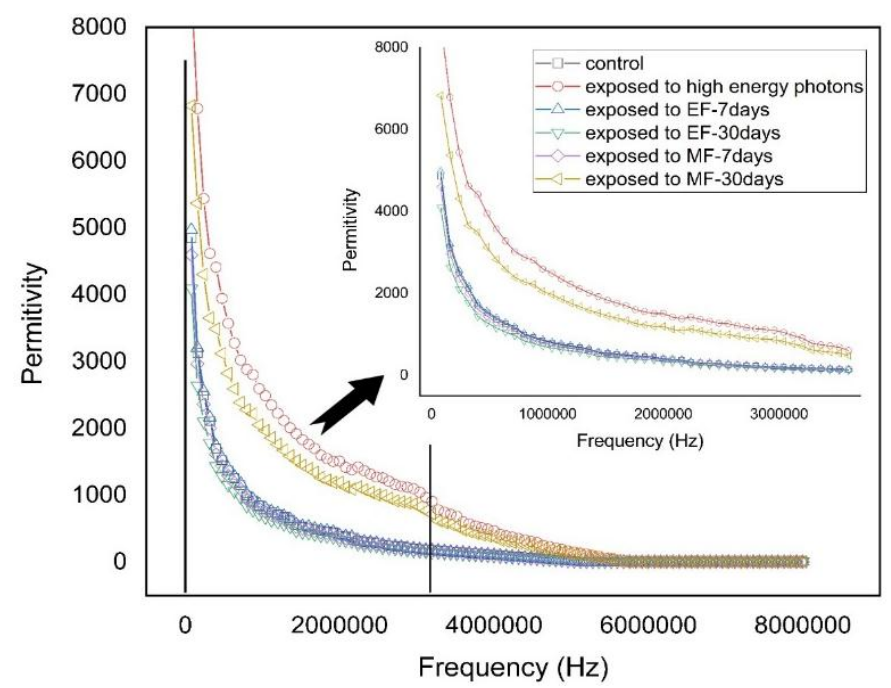

Fig. (4): The relative permittivity relaxation curves of blood samples collected after 45-days post exposure
It should be born in mind, the blood cells conductivity shows high sensitivity to the radiations and the presence of hemoglobin molecules increase such effect. Also, any damage in cellular permeability and loss of cell membrane integrity attributed to the exposure may lead to subsequent loss of intracellular components, electrolytes and ions and as a result, the cell conductivity will be changed. [14]

The dielectric loss of blood cells is an electro-metric sensor of the responsive action of molecules under the application of different frequencies and its energy loss relative to the original molecular sites. Figure (5) shows the dielectric loss relaxation curves attributed to the exposure in comparison to the control ones. The obtained relaxations showed same dispersion over all the examiner frequency range for all tested samples but with different orders. The order of dispersions is clearly observed at the frequency range less than $4 \mathrm{MHz}$, as depicted in the magnified upper side. The obtained dispersions showed a maximum shift to lower values for samples exposed to MF-30 days and followed by samples exposed to high energy photons in resemblance to the results of the conductivity and relative permittivity. On contrary, the obtained curves showed no difference between control and exposed samples to EF-7 days whereas the exposure to EF-30 days showed a significant shift to low values to come closer to the samples exposed to high energy photons.

Similar to the former discussed results of conductivity and relative permittivity of samples collected after 45-days post exposure; the data of dielectric loss didn't show enhancement of blood bioelectricity to return back to its original normal conditions. Figure (6) shows the dielectric loss dispersions for samples collected after 45 days' post exposure and it depicted a maximum remnant drop in samples exposed to MF-30days and samples exposed to high energy photons. The relaxations dielectric parameters of blood cells are directly related to polarizability of cellular constituents under external field application in a way it forms electrical dipole moments. Such dipole moments are dependent on two mainly factors which are size and charge carried by macromolecules implemented in blood cellular envelope. In this regard the scenario of low frequency shifts ( $\alpha$-dispersions), could be illustrated based on the hypothesis of the counterion polarization imbedded from blood cellular membrane that performed its external charges. To this end, the alterations in the dielectric characteristics ascribed to the relaxation, in a direction tangential to the cellular surface, of the ion cloud constituting the diffuse double layer surrounding it. Taking into account the wide range of macromolecules dimensions bounded to the membrane and a variety of its charge distribution, it shows broad relaxation times in response to the applied external fields [21-25]. Moreover, the charge and current densities induced in response to an applied external field of unit amplitude it changes the conductivity and permittivity of a sample. So, any structural changes and charge dislocation may be attributed to the exposure and will be accompanied with remarkable changes in the dielectric sample characteristics. According to the applied frequency range; at very low 
frequencies, the volume fraction of extracellular fluids affects strongly the conductivity while in frequency range from $1 \mathrm{MHz}$ up to $100 \mathrm{MHz}$ the cell membrane is largely shorted out and do not provide a significant barrier to

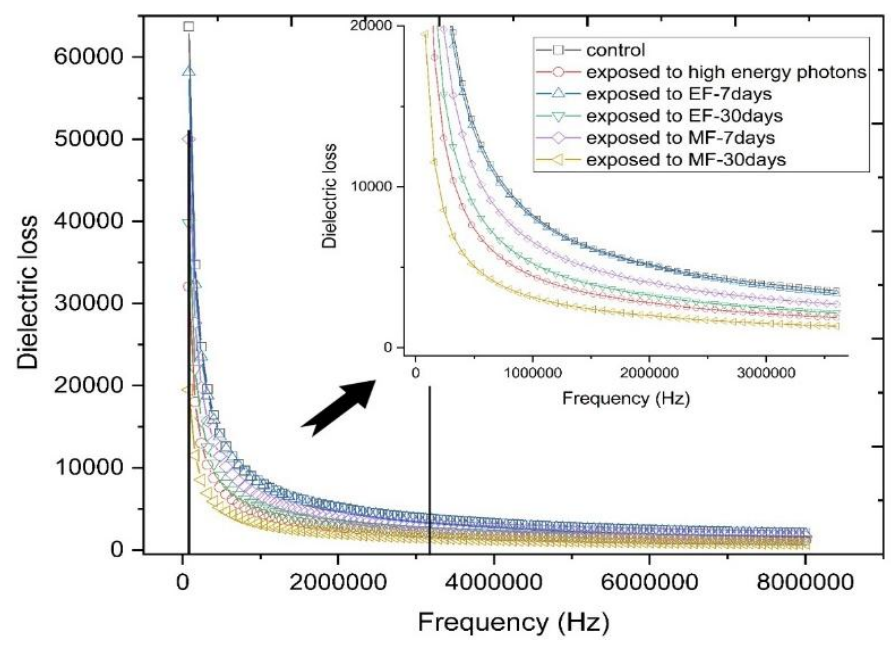

Fig. (5): The dielectric loss relaxation curves of blood samples collected directly after exposure current flow. In this regard, the blood biochemical concentrations were measured for samples collected directly after exposure and after 45 days' post exposure as listed in Tables (1 and 2) respectively.

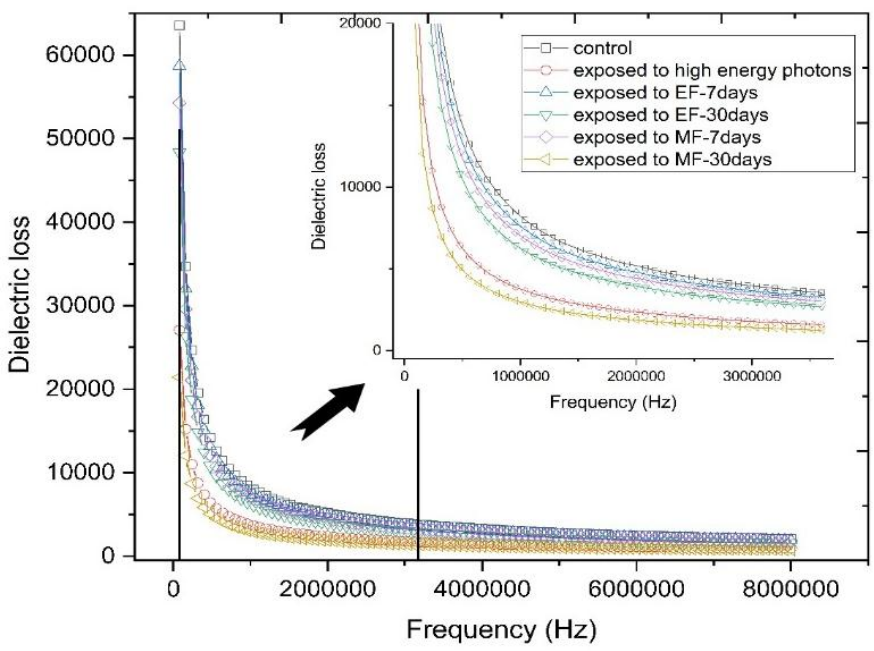

Fig (6): The dielectric loss relaxation curves of blood samples collected after 45-days post exposure

Table (1): The concentrations of some biochemical parameters of blood samples collected directly after exposure

\begin{tabular}{|c|c|c|c|c|c|}
\hline Groups & $\begin{array}{c}\text { sodium } \\
(\mathbf{m m o l} / \mathrm{l})\end{array}$ & $\begin{array}{c}\text { calcium } \\
(\mathrm{mmol} / \mathrm{l})\end{array}$ & $\begin{array}{c}\text { phosphates } \\
(\mathbf{m m o l} / \mathrm{l})\end{array}$ & $\begin{array}{c}\text { potassium } \\
(\mathbf{m m o l} / \mathrm{l})\end{array}$ & $\operatorname{protein}(g / \mathbf{l})$ \\
\hline Control & $136.2 \pm 0.06$ & $2.83 \pm 0.17$ & $2.24 \pm 0.08$ & $5.82 \pm 0.09$ & $57.72 \pm 0.32$ \\
\hline Exposed to high energy photons & $136.6 \pm 0.12$ & $2.34 \pm 0.11 *$ & $2.55 \pm 0.06 *$ & $6.12 \pm 0.13 *$ & $54.61 \pm 0.24 * *$ \\
\hline Exposed to MF-7days & $136.3 \pm 0.09 *$ & $2.77 \pm 0.13$ & $2.31 \pm 0.11 *$ & $5.94 \pm 0.18$ & $56.88 \pm 0.25 *$ \\
\hline Exposed to MF-30days & $136.4 \pm 0.13 *$ & $2.68 \pm 0.12 * *$ & $2.38 \pm 0.09 * *$ & $5.98 \pm 0.09 *$ & $55.92 \pm 0.36$ \\
\hline Exposed to EF-7days & $136.3 \pm 0.07$ & $2.81 \pm 0.12$ & $2.29 \pm 0.09$ & $5.88 \pm 0.19 *$ & $57.08 \pm 0.45 *$ \\
\hline Exposed to EF-30days & $136.3 \pm 0.06 * *$ & $2.79 \pm 0.14 *$ & $2.33 \pm 0.04 *$ & $5.91 \pm 0.16$ & $56.91 \pm 0.37 * *$ \\
\hline
\end{tabular}

* Statistically significant

** Statistically highly significant

Table (2): The concentrations of some biochemical parameters of blood samples collected after 45-days post exposure

\begin{tabular}{|c|c|c|c|c|c|}
\hline Groups & $\begin{array}{l}\text { sodium } \\
(\mathrm{mmol} / \mathrm{l})\end{array}$ & $\begin{array}{l}\text { calcium } \\
(\mathrm{mmol} / \mathrm{l})\end{array}$ & $\begin{array}{c}\text { phosphates } \\
(\mathrm{mmol} / \mathrm{l})\end{array}$ & $\begin{array}{c}\text { potassium } \\
(\mathrm{mmol} / \mathrm{l})\end{array}$ & protein $(g / l)$ \\
\hline Control & $136.2 \pm 0.07$ & $2.83 \pm 0.18$ & $2.24 \pm 0.09$ & $5.82 \pm 0.10$ & $57.72 \pm 0.33$ \\
\hline Exposed to high energy photons & $136.5 \pm 0.13 * *$ & $2.64 \pm 0.12$ & $2.35 \pm 0.07 *$ & $6.02 \pm 0.14$ & $56.91 \pm 0.24 *$ \\
\hline Exposed to MF-7days & $136.2 \pm 0.10$ & $2.8 \pm 0.14 *$ & $2.27 \pm 0.12 * *$ & $5.84 \pm 0.19$ & $57.11 \pm 0.26 *$ \\
\hline Exposed to MF-30days & $136.3 \pm 0.14 *$ & $2.79 \pm 0.13$ & $2.28 \pm 0.10 *$ & $5.88 \pm 0.10 * *$ & $57.02 \pm 0.37$ \\
\hline Exposed to EF-7days & $136.2 \pm 0.08$ & $2.82 \pm 0.13 *$ & $2.24 \pm 0.10$ & $5.84 \pm 0.20$ & $57.58 \pm 0.46$ \\
\hline Exposed to EF-30days & $136.2 \pm 0.07 * *$ & $2.81 \pm 0.15$ & $2.26 \pm 0.05 * *$ & $5.87 \pm 0.17$ & $57.51 \pm 0.38 * *$ \\
\hline
\end{tabular}

* Statistically significant

** Statistically highly significant 
The obtained concentration data of the biochemical parameters supported that the changes in thedielectric properties due to exposure might have resulted from the alterations in the ionic concentrations on cellular both sides. Such structural alterations could be related to the ionic pump dysfunctionality and modification of ionic gradient [26-29]. The ability of EF and MF to change the dipole's orientation of the membrane components led to changing the charge allocations across the cell membrane [30-32]. As a result of charge redistribution the enzymatic receptors were altered and the ionic concentrations across the membrane were changed. Particularly, the former findings proved that the exposure to magnetic field has a higher effect than the electric filed in manner that it may cause permanent deteriorated effect. In comparison to the control samples, the change of conductivity for samples collected directly after exposure to MF-30 days and high energy photons showed maximum percentages at $5 \mathrm{MHz}$ by $62 \%$ and $46 \%$ respectively. Similarly, without remarkable recovery the conductivity for samples collected 45 days' post exposure showed $60 \%$ and $45 \%$ of changes after exposure to MF-30 days and high energy photons. In the context the permittivity results supported the maximum changes at $5 \mathrm{MHz}$ attributed to the exposure of MF-30 days and high energy photons that it became higher than control by 23 and 8 folds respectively for samples collected directly and by 10 and 8 folds for samples collected delayed after exposure. Moreover, the ability of external electromagnetic fields to interact with bioelectric signals of blood cells might be the main reason of the cellular alterations resulted from the exposure. One may notice the direct effect of ionizing radiation on the minerals concentration of blood as it indicated percentage of change in relative to control by $13 \%, 17 \%$ and $5 \%$ for phosphate, calcium and potassium respectively. In addition, the minerals concentration showed resemblance to the dielectric characteristics as concentrations of phosphate, calcium and potassium changed by $6 \%, 5 \%$ and $3 \%$ respectively for samples collected after exposure to MF-30 days. The variation of the effect of electric, magnetic fields on blood minerals may be due to the charge amounts to mass of each element whereas the fields may interact to accelerate the charges (as electric field influences) and/or reorient its directions (as magnetic field influences). The changes in the dielectric parameters proved the possible ionic permeability alterations attributed to the exposure of EF and MF are noticeable in comparison with exposure to high energy photons. In addition, the exposure effect is dependent on the period of exposure and type of field in addition to the field strength.

\section{CONCLUSION}

The present findings illuminated the significant alterations in the dielectric characteristics and biochemical analysis of blood samples after exposure to the proposed fields. The exposure effects are dependent on the exposure time and the filed type in a manner that exposure to magnetic field has higher effect than exposure to electric field even for same exposure periods. is the findings of the present study suggest that same concerns should be considered regarding the effect of exposure to non-ionizing radiations as ionizing radiation, especially for prolonged exposure periods. Full blood analysis for exposed persons should be done frequently and to include dielectric characterization as a parameter that reflects the possible cellular injuries. There is a need for more work to be done for examining the possible health consequences due to exposure to such nonionizing radiations and to set new exposure limits.

\section{REFERANCES}

[1] Li Gun, Du Ning., (2017), "Equivalent Permittivity Based on Debye Model of Blood and Its SAR", International Journal of Science, Technology and Society, doi: 10.11648/j.ijsts.20170503.12 ISSN: 2330-7412 (Print); ISSN: 2330-7420 (Online); pp.5(3):37-40.

http://www.sciencepublishinggroup.com/j/jijsts.

[2] J. Leroy, C. Dalmay, A. Landoulsi., (2015) "Microfluidic biosensors for microwave dielectric spectroscopy," Sensors and Actuators, A: Physical, vol. 229, pp. 172-181.

[3] Y. Otaki, Y. Ebana, S. Yoshikawa, and M. Isobe, (2016) "Dielectric permittivity change detects the process of blood coagulation: comparative study of dielectric coagulometry with rotational thromboelastometry," Thrombosis Research, vol. 145, pp. 3-11.

[4] Walter R.J., A.A. Shtil, R.B. Roninson, D. Halian, (1997) "60 Hz electric fields inhibit protein kinase $\mathrm{C}$ activity and multidrug resistance gene (MDRI) up-regulation, Rad. Res. Mar., 147 (3), 369-382.

[5] M. SAID, (2006), "Electric field affected the molecular structure of the total serum proteins of the mice blood," Romanian J. Biohys., Vol. 16, No. 3, P. 205-214, BUCHAREST.

[6] MAGDY, M., R.H. GHANNAM, M.H. ELGEBALY, GABER, F. M. ALI, (2002) "Inhibition of ehrlich tumor growth in mice by electric interference therapy (in vivo studies)", Electromagnetic biology and medicine, , 21, 255268. 
[7] HASTED, JB., (1973) 'Aqueous Dielectrics', Chapman and Hall Ltd, eds., London.

[8] MCCAMMON, J.A., S.C. HARVEY, (1987) 'Dynamics of Proteins and Nucleic Acids', Cambridge, Cambridge University Press, London.

[9] Calabrò, E.; Condello, S.; Currò, M.; Ferlazzo, N.; Vecchio, M.; Caccamo, D.; Magazù, S.; Ientile, R., (2013) " $50 \mathrm{~Hz}$ electromagnetic field produced changes in FTIR spectroscopy associated with mitochondrial transmembrane potential reduction in neuronal-like SH-SY5Y cells". Oxid. Med. Cell. Longev., 414393.

[10] Ye, H.; Cotic, M.; Kang, E.E.; Fehlings, M.G.; Carlen, P.L. (2010) "Transmembrane potential induced on the internal organelle by a timevarying magnetic field: A model study". J. Neuroeng. Rehabil., 7, 12.

[11] Liu, Y.; Weng, E.; Zhang, Y.; Hong, R., (2002) "Effects of extremely low frequency electromagnetic field and its combination with lead on the antioxidant system in mouse". Zhonghua Lao Dong Wei Sheng Zhi Ye Bing Za Zhi, 20, 263-265. Available online: https://www.ncbi.nlm.nih.gov/pubmed/1469464.

[12] Mai. I. El-kaliuoby, (2019)," A comparative Study Between the Exposures of $6 \mathrm{MeV}$ Photons and $0.3 \mathrm{mT}-50 \mathrm{~Hz}$ Magnetic Fields in Rat's Liver". Arab J. Nucl. Sci. Appl., Vol. 52, 3, 249258.

[13] Zhang, X.; Liu, X.; Pan, L.; Lee, I., (2010), "Magnetic fields at extremely low-frequency (50 $\mathrm{Hz}, 0.8 \mathrm{mT}$ ) can induce the uptake of intracellular calcium levels in osteoblasts". Biochem. Biophys. Res. Commun. 396, 662-666.

[14] Nabila S. Selim., O.S. Desouky., Seham M. Ali., I.H. Ibrahim and Hoda A. Ashry., (2009), "Effect of gamma radiation on some biophysical properties of red blood cell membrane". Romanian J. Biophys., Vol. 19, No. 3, P. 171185, Bucharest.

[15] Nabila S. Selim, (2010), "Comparative study on the effect of radiation on whole blood and isolated red blood cell". Romanian J. Biophys., Vol. 20, No. 2, P. 127-136, Bucharest.

[16] S.H. Allehyani, H. S. Ibrahim, F. M. Ali, E. Sayd and T. Abou Aiad, (2016), "In vivo spectroscopic study on the effects of ionizing and non-ionizing radiation on some biophysical properties on rat blood". Journal of Applied Medical Sciences, vol.5, no. 1, 2016, 65-80 ISSN: 2241-2328 (print version), 2241-2336.

[17] Soliman M., (2004), "Whole body gamma radiation effects on rheological behavior (deformability of rat erythrocytes)". Egypt. J. Rad. Sci. Applic., 17 (2), 345-363.

[18] Alaa M. Khalil, (2019), "Relative Biophysical Effects on Rat's Bone as a Result of High Energy Photons and Electromagnetic Fields Exposures. Arab J. Nucl. Sci. Appl., 52(4). 21-27.

[19] R. J. Henry; D. C Cannon; J. W Winkelman; (1974), Clinical chemistry: principles and techniques; Hagerstown, Maryland: Harper and Row.

[20] O H Lowry, N J Rosebrough, A L Farr, R J Randall, (1951) Protein measurement with the Folin phenol reagent J Biol Chem;193(1):265-75.

[21] Charles Polk and Elliot Postow., (1995), 'Handbook of Biological Effects of Electromagnetic Fields, part-I', Published by CRC Press, ISBN 10: 0849306418.

[22] Mai. I. El-kaliuoby, Alaa M. Khalil and Ahmed. M. El-Khatib, (2020) Alterations of bacterial dielectric characteristics due to pulsed magnetic field exposure. Bioinspired, Biomimetic and Nanobiomaterials $\quad 9(2): \quad 103-111$, https://doi.org/10.1680/jbibn.19.00055

[23] Mai I Elkaliuoby, Ahmed M El-Khatib and Alaa M Khalil (2019). Does Engineering of Nano Shapes Have Antibacterial Synergy with Magnetic Signals Exposure? Surface Innovations, 7(5), 260-267.

[24] Ahmed M El-Khatib, Alaa M Khalil, Mai I Elkaliuoby, Mostafa Elkhatib. (2019). The combined effects of multisized silver nanoparticles and pulsed magnetic field on $\mathrm{K}$. pneumoniae. Bioinspired, Biomimetic and Nanobiomaterials, 8(2), pp. 154-160.

[25] Mai I Elkaliuoby, Alaa M Khalil, Ahmed M ElKhatib and Thanaa I. Shalaby (2018) Synergistic antibacterial effect of silver nanoparticles and extremely low frequency pulsed magnetic fields on Klebsiella pneumoniae. Journal of Applied Biology \& Biotechnology 6(6): 039-045.

[26] Mai. I. El-kaliuoby, Ahmed. M. El-Khatib, Khalil, A. M, Mohamed Ahmed Abbas Naim and Malak M. Elmesady. (2017) Monitoring of relative changes in rats' tibia bone characteristics after 
exposure to $4 \mathrm{kv} / \mathrm{m}-50 \mathrm{hz}$ electric fields", International Journal of Current Research, 9, (12), 62026- 62031.

[27] Alaa M. Khalil, Ahmed. M. El-Khatib, Moustafa M. Mohamed, Mostafa El-Khatib Hanan. M. Abdulsalam, Gamal D Roston (2016) "Monitoring of Variations in Some Hematological Parameters and Liver Enzymes as a Result of Exposure to 50$\mathrm{Hz}$ Electric Fields" J. Harmoniz. Res. Med. and Hlth. Sci., 3(2), 113-120.

[28] Fadel M.Ali, Osoris W.G, N.Serag, A. M. Khalil. (2016); "Healing of Guinea Pig Injures Contaminated with Pseudomonas aeruginosa by using $0.7 \mathrm{~Hz}$ Square Pulsed Magnetic Field (New Method)" Int. J. Curr. Res. Med. Sci. 2(5): 6-11.

[29] Fadel M. Ali, A. M. Elkhatib, W. M. Aboutaleb, A. M. Abdelbacki, A. M. Khalil and Mai. I. Elkaliuoby. (2014) "Control the Activity of Ralstonia Solanacearum Bacteria by Using Pulsed
Electric Field" Jokull Journal Vol 64, No. 4; (255269).

[30] Fadel M. Ali, A. M. Elkhatib, W. M. Aboutalib, A. M. Abdelbacki, A. M. Khalil, and N.Serag. (2013) "Control of the Activity of Pseudomonas Aeruginosa by Positive Electric Impulses at Resonance Frequency" J Am Sci; 9(10):120-130.

[31] Naglaa M Balabel, Mai I El-Kaliuoby and Alaa M Khalil (2019). Effect of square pulsed magnetic field exposure on growth kinetics of Dickeya solani. Archives of Phytopathology and Plant Protection 52:11-12, 989-1004.

[32] Cieślar, G., Sieroń, A., Turczyński, B., Adamek, M., \& Jaskólski, F. (1994). The influence of extremely low-frequency variable magnetic fields on rheologic and dielectric properties of blood and the water-electrolyte balance in experimental animals. Bioelectrochemistry and Bioenergetics, 35(1-2),29-32. doi:10.1016/0302-4598(94)87007-1. 